\title{
Neurofibroma plexiforme gigante da região lombar com transformação hemorrágica - Relato de caso
}

\author{
Mayara Dalila Cardoso de Lima', Washington Luiz de Oliveira ${ }^{1}$, Carlos \\ Elizeu Barcelos ${ }^{1}$, Sergio Luiz Sprengel2 ${ }^{\text {, João Cândido Araújo }}{ }^{3}$ \\ Hospital de Clínicas da Universidade Federal do Paraná (HC-UFPR), Curitiba, PR, Brasil.
}

\section{RESUMO}

A neurofibromatose tipo 1 é uma facomatose que apresenta, entre outras características, tumores oriundos da bainha dos nervos como o neurofibroma plexiforme. Neste artigo é relatado o caso de um paciente que sofreu transformação hemorrágica espontânea de um neurofibroma plexiforme gigante na região lombar. Existem apenas cinco casos relatados na literatura de neurofibroma plexiforme gigante na região lombar, havendo apenas dois casos de transformação hemorrágica de tal lesão, sendo este o terceiro maior neurofibroma plexiforme relatado na região lombar. O paciente foi submetido à cirurgia e à ressecção total da lesão. O anatomopatológico confirmou o diagnóstico de neurofibroma plexiforme e revelou a presença de vasos displásicos, que poderiam ser a causa do sangramento espontâneo. É ressaltada a necessidade de orientação aos pacientes quanto ao crescimento súbito da lesão e à hemorragia como diagnóstico diferencial etiológico de tal crescimento.

\section{PALAVRAS-CHAVE}

Neurofibromatose 1, neurofibroma plexiforme, hemorragia.

\begin{abstract}
Giant plexiform neurofibroma of the lumbar region with hemorrhagic transformation - Case report

Neurofibromatosis type 1 is a phakomatose that may present as a variant called plexiform neurofibroma. The case of a patient who suffered a spontaneous hemorrhagic transformation of a giant plexiform neurofibroma in the lumbar region is discussed. There are only five cases reported of giant plexiform neurofibroma in the lumbar region and two cases of hemorrhagic transformation of this type of tumor. The lesion was totally resected and is the third largest plexiform neurofibroma reported. The histopathological exam confirmed the diagnosis and revealed the presence of dysplastic vessels, the probable cause of spontaneous bleeding. The authors emphasize the need of orientation to the patients about the possibility of fast growth of the lesion due to spontaneous bleeding.
\end{abstract}

\section{KEYWORDS}

Neurofibromatosis 1, neurofibroma plexiform, hemorrhage.

1 Residente do Serviço de Residência Médica em Neurocirurgia do Hospital das Clínicas da Universidade Federal do Paraná (HC-UFPR), Curitiba, PR, Brasil.

2 Neurocirurgião do Serviço de Residência Médica em Neurocirurgia do HC-UFPR, Curitiba, PR, Brasil.

3 Chefe do Serviço de Residência Médica em Neurocirurgia do HC-UFPR, Curitiba, PR, Brasil. 


\section{Introdução}

A neurofibromatose 1 (NF1) é uma facomatose autossômica dominante com amplo espectro de manifestações clínicas e tem como característica principal a presença de tumores derivados da crista neural ${ }^{1}$ que incluem o neurofibroma plexiforme (NP). Este constitui um tumor benigno da bainha dos nervos periféricos, cuja presença é um dos critérios diagnósticos de NF1. Apesar de apresentar crescimento lento, tal lesão pode atingir tamanho considerável como 70 x 80 centímetros. $^{2} \mathrm{O}$ tumor relatado neste artigo apresentava $20 \times 13 \times 10$ centímetros, sendo o terceiro maior NP lombar relatado, podendo provocar déficit ou desfiguramento. Degeneração maligna ocorre em 2\% a $5 \%$ dos casos. ${ }^{2,3}$ Há apenas dois relatos de hemorragia associada ao NP lombar na literatura. O caso de um paciente que apresentou um neurofibroma plexiforme lombar com hemorragia como causa do crescimento rápido da lesão é relatado neste artigo.

\section{História clínica}

Um paciente do sexo masculino de 30 anos deu entrada no serviço de pronto atendimento com queixa de aumento rapidamente progressivo de tumor em região lombar nas duas semanas anteriores e dificuldade respiratória (Figura 1A e B). Ele já apresentava tal lesão há três anos e havia recebido o diagnóstico de neurofibromatose tipo 1 e pneumopatia restritiva, devido à cifoescoliose acentuada da coluna torácica, dois meses antes desse internamento. O paciente já estava restrito à cadeira de rodas, além de apresentar incontinência urofecal e nível sensitivo em T10 havia quatro anos, cuja causa não foi determinada após extensa investigação com ressonância nuclear magnética (RNM) e arteriografia. Ao exame apresentava manchas café com leite, vários nódulos subcutâneos de pequeno tamanho e um grande tumor em região lombar, sem alterações na pigmentação da pele sobre a lesão. A RNM de coluna lombar mostrou volumosa formação expansiva loculada de conteúdo misto, localizada entre o tecido subcutâneo e a musculatura lombar, estendendo-se desde o nível de L1/L2 ao sacro, medindo em torno de $20 \times 13 \times 10 \mathrm{~cm}$ com realce pelo contraste paramagnético em T1 e hipersinal em T1 e T2. A lesão não se mostrava contínua com o canal vertebral, mas havia disrafismo sacral (Figura 2A e B). Os exames laboratoriais incluindo hemograma e coagulograma estavam dentro da normalidade. $\mathrm{O}$ paciente foi então submetido à cirurgia com ressecção de toda a lesão que se encontrava abaixo do subcutâneo e acima dos planos musculares com limites bem definidos e seu interior completamente preenchido por hematoma liquefeito e coágulos (Figuras 3 e 4). O sangramento durante o procedimento não foi significativo. $O$ paciente recebeu alta hospitalar cerca de três semanas depois, após estabilização de sua condição respiratória, uma vez que ele apresentou piora transitória da pneumopatia restritiva no pós-operatório. A anatomia patológica mostrou tratar-se de lesão contendo proliferação celular composta por células fusiformes com núcleos alongados envoltas por matriz colágena e células fusiformes com núcleos ovoides ou curvados, ambos sem atipias, observando-se também vasos ectásicos com paredes delgadas compostas, por vezes apenas por endotélio. A lesão foi compatível com neurofibroma plexiforme com extensa hemorragia recente.
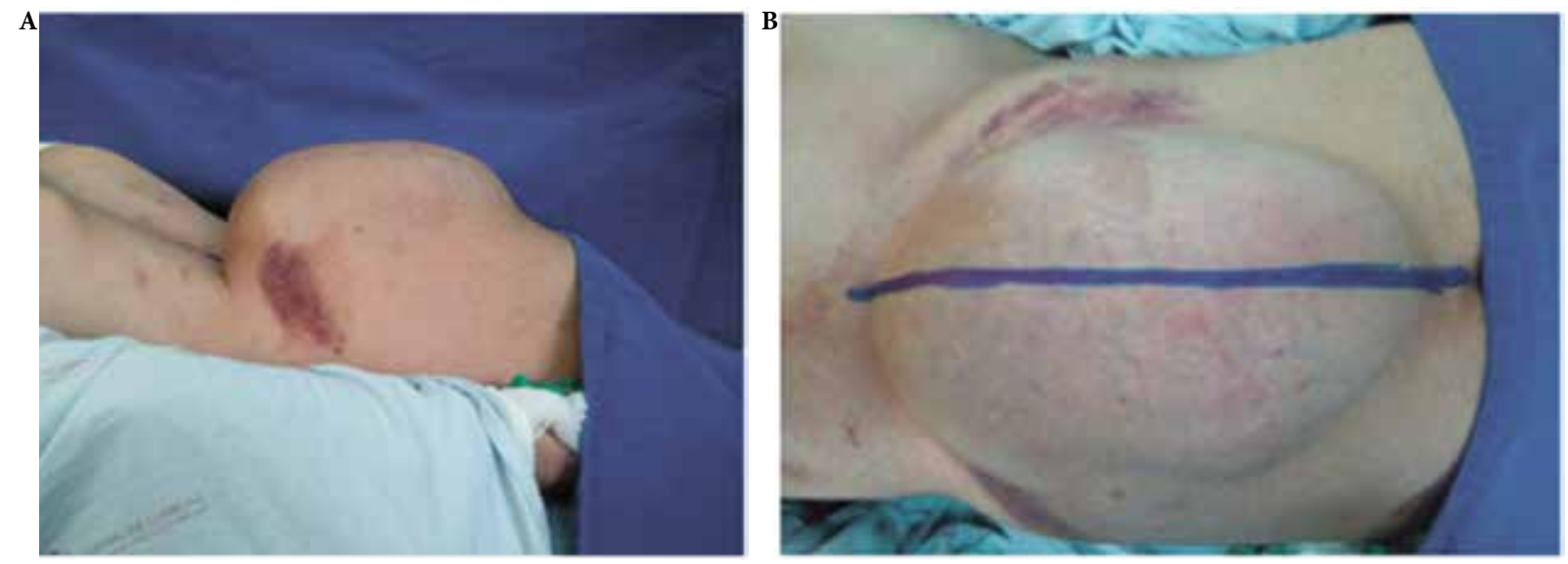

Figura 1 - (A) Foto pré-operatória de perfil do paciente em decúbito ventral mostrando a lesão em região lombar. (B) Foto pré-operatória em incidência póstero-anterior do paciente em decúbito ventral. 

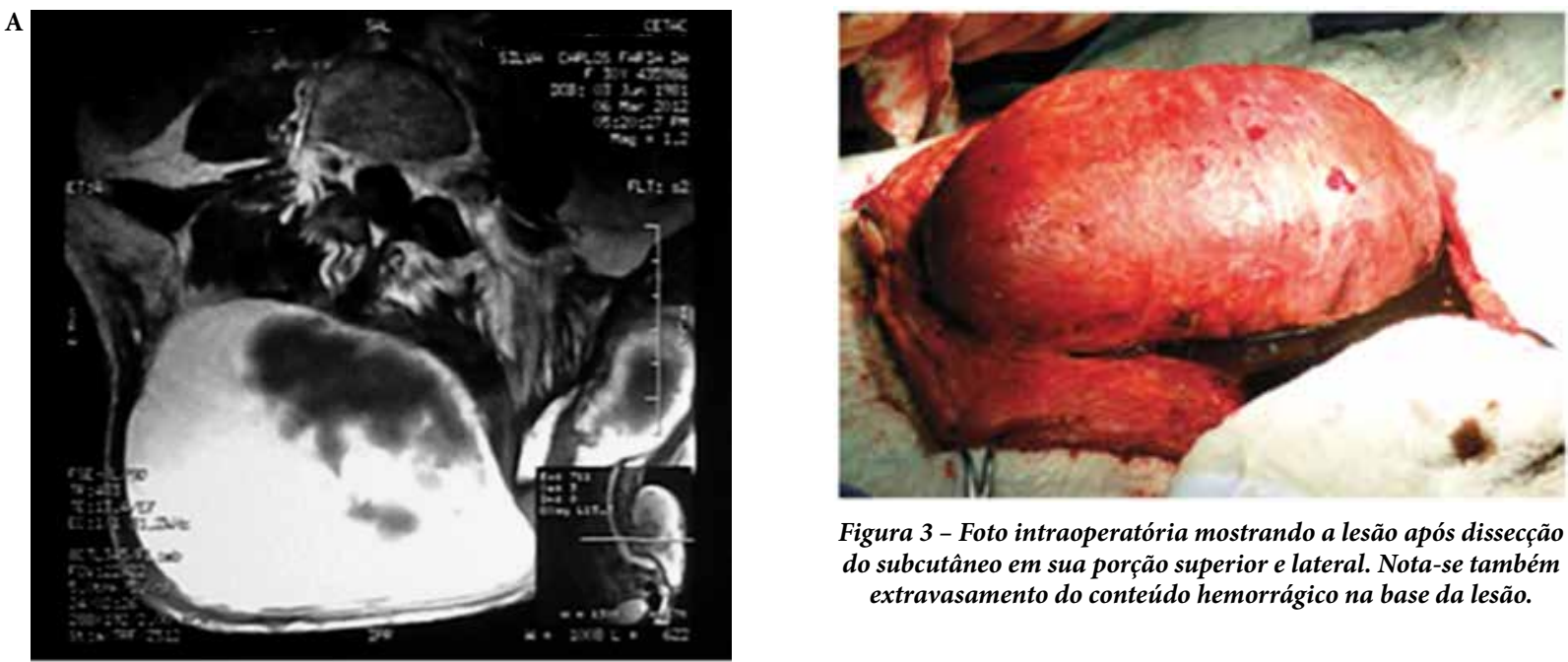

Figura 3 - Foto intraoperatória mostrando a lesão após dissecção do subcutâneo em sua porção superior e lateral. Nota-se também extravasamento do conteúdo hemorrágico na base da lesão.

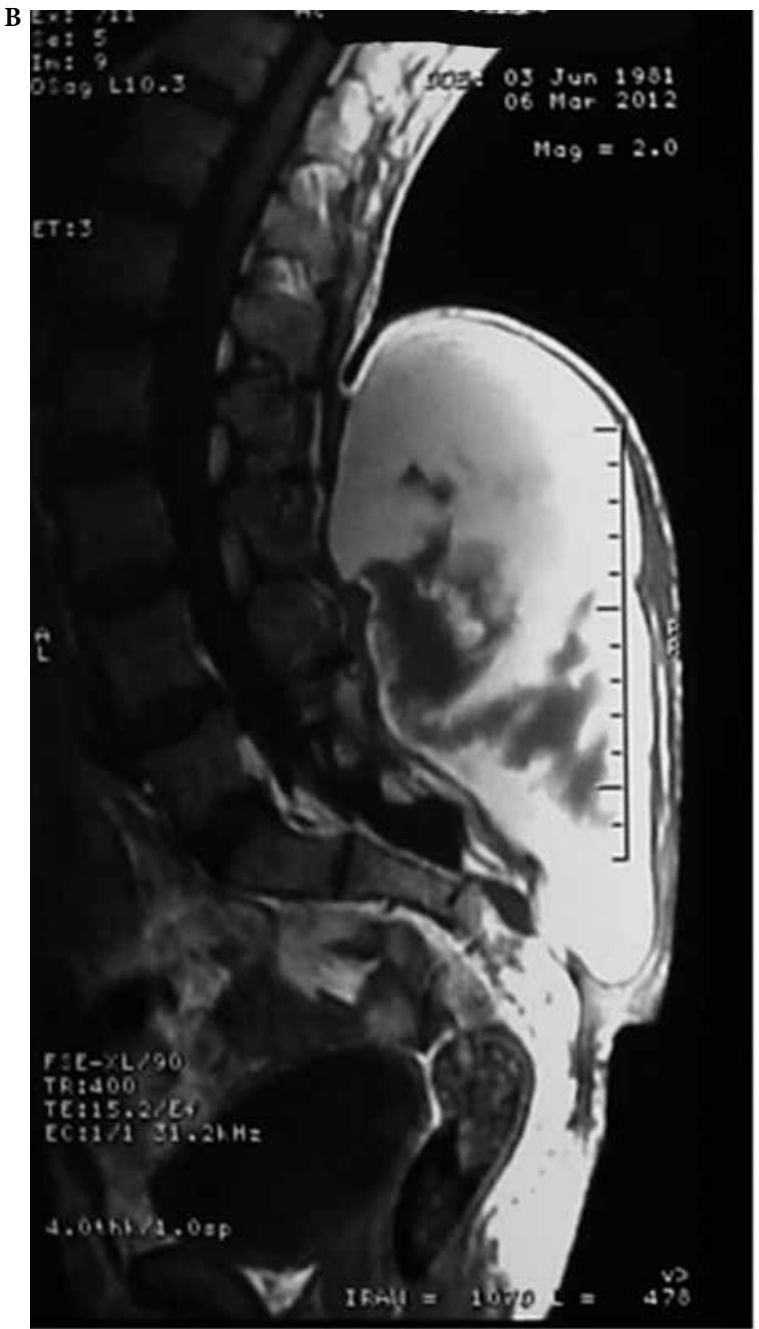

Figura 2 - (A) RNM ponderada em T2 axial mostrando lesão hiperintensa subcutânea, sem continuidade com o canal vertebral. (B) RNM ponderada em T1 sagital mostrando lesão hiperintensa sem comunicação com o canal vertebral.

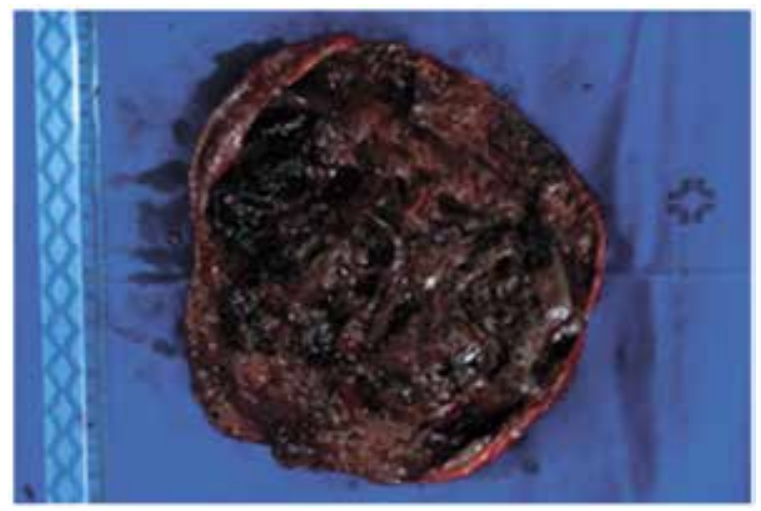

Figura 4 - Imagem mostrando a lesão com seu conteúdo composto de hematomas.

\section{Discussão}

A NF tipo 1 é causada por uma mutação no gene que codifica a neurofibromina, o qual se localiza no braço longo do cromossomo 17.4 A neurofibromina está presente em neurônios, oligodendrócitos e células de Schwann. Sua principal função é a de inativar a enzima RAS-GTP e suas vias de transdução de sinal. No entanto, com a mutação, essa função é perdida, o que poderia levar a proliferação celular e formação de neurofibromas. ${ }^{1}$ A penetrância dessa mutação é completa. ${ }^{4}$

O NP constitui uma característica cardinal da NF1, com uma prevalência de $44 \% .^{5}$ Esses tumores são formados por uma variedade de células como as células de Schwann, fibroblastos, mastócitos, células endoteliais e células de músculo liso e crescem ao longo de um determinado nervo, podendo englobar múltiplos 
fascículos e situar-se em qualquer camada tecidual. ${ }^{1,6}$ Podem ser localmente invasivos, porém não cursam com metástase. ${ }^{3} \mathrm{O}$ surgimento costuma ocorrer durante a infância, ${ }^{4}$ porém o paciente em questão relatou o aparecimento da lesão, de forma gradual, aos 27 anos de idade.

Essas lesões são encontradas principalmente no tronco, podendo ser superficiais, quando afetam pele ou subcutâneo, ou profundas quando acometem as vísceras. Entretanto, podem surgir em qualquer segmento corpóreo e apresentam crescimento lento ao longo dos anos., ${ }^{4,7}$ Não há tratamento específico para o NP e a ressecção total é recomendada em casos de dor, incapacidade funcional ou deformidade. A cirurgia também está indicada em casos de uma súbita aceleração de sua taxa de crescimento devido à possibilidade de degeneração maligna em neurofibrossarcoma, neurossarcoma e Schwannoma maligno. ${ }^{3,5}$ Essa possibilidade torna mandatório o acompanhamento periódico de lesões preexistentes e orientação aos pacientes.

No caso relatado, houve um grande aumento da lesão em região lombar em apenas duas semanas, associado ao fato de este apresentar hiperintensidade de sinal nas sequências ponderadas em T1 e T2 no seu interior, o que sugeria tratar-se de uma hemorragia subaguda. A ocorrência de hemorragia como causa de crescimento rápido de um neurofibroma plexiforme lombar é rara, havendo apenas dois casos relatados na literatura.,8

O procedimento cirúrgico para ressecção tumoral pode ser difícil devido ao tamanho e à localização, principalmente nos casos de lesões situadas profundamente. $\mathrm{O}$ paciente do caso em questão apresentava o NP logo abaixo do tecido subcutâneo, o que acarretou a vantagem de haver um plano de clivagem bem definido possibilitando sua retirada completa. Deve ser realizada uma hemostasia criteriosa durante a cirurgia, uma vez que o NP é uma lesão hipervascularizada e sangramentos de grande intensidade podem ocorrer durante ou após o ato cirúrgico. ${ }^{7}$

A causa do sangramento associado ao neurofibroma pode estar relacionada aos vasos ectásicos e displásicos evidenciados na análise histopatológica, uma vez que o paciente apresentava coagulograma normal, o que é compatível com a literatura. ${ }^{7}$

Neste relato é apresentado um caso de NP gigante com transformação hemorrágica. Existem apenas cinco casos de NP gigante lombar descritos, sendo dois associados com sangramento. Essa possibilidade etiológica deve entrar no diagnóstico diferencial de lesões de grande volume que cursam com crescimento rápido.

\section{Conflito de interesses}

Os autores declaram não haver conflito de interesses na realização deste trabalho.

\section{Referências}

1. Gottfried ON, Viskochil DH, Fults DW, Couldwell WT. Molecular, genetic, and cellular pathogenesis of neurofibromas and surgical implications. Neurosurgery. 2006;58(1):1-16.

2. Ritz GM, Bez Batti HT, Vigeti NC, Roça GB, Pintarelli G. Neurofibroma plexiforme gigante de dorso - Relato de caso. Arq Catarin Med. 2009;38(01):67-9.

3. Serletis D, Parkin P, Bouffet F, Shroff M, Drake JM, Rutka JT. Massive plexiforme neurofibromas in childhood: natural history and management issues. $J$ Neurosurg. 2007;106(5):363-7.

4. Sehgal VN, Srivastava G, Aggarwal AK, Oberai R. Plexiform neurofibromas in neurofibromatosis type 1. Int J Dermatol. 2009;48(9):971-4.

5. Washington EN, Placket TP, Gagliano RA, Kavolius J, Person DA. Diffuse plexiform neurofibroma of the back: report of a case. Hawaii Med J. 2010;69(8):191-3.

6. Feng Y, Yang Z, Chen T, Wang Q, Deng W. Giant plexiform neurofibroma with hemorrhage in cranio-maxillofacial region as depicted on CT and MRI. Eur J Med Res. 2010;15(2):84-7.

7. Yang L, Robertson T, Tollesson G, Francis L, Campbell $D$, Winter C. An unusual presentation of a solitary benign giant neurofibroma. J Neurosurg Spine. 2009;11(1):49-52.

8. Daher S, Sousa Junior ZA, Mendonça MI, Pimenta Junior WE, Silveira SAR, Moraes FB. Neurofibroma lombar gigante. Coluna/Columna. 2002;1(2):103-6.

Endereço para correspondência

Mayara Dalila Cardoso de Lima

Rua Ubaldino do Amaral, 360, ap. 28

80060-190 - Curitiba, PR, Brasil

Telefone: (41) 9928-2878

E-mail: mayaradalila@hotmail.com 